\title{
Dlx-2 and glutaminase upregulate epithelial-mesenchymal transition and glycolytic switch
}

\author{
Su Yeon Lee ${ }^{1}$, Hyun Min Jeon ${ }^{1}$, Min Kyung Ju ${ }^{1}$, Eui Kyong Jeong ${ }^{1}$, Cho Hee Kim ${ }^{1, *}$, \\ Hye Gyeong Park ${ }^{2}$, Song Iy Han ${ }^{3}$, Ho Sung Kang ${ }^{1}$ \\ ${ }^{1}$ Department of Molecular Biology, College of Natural Sciences, Pusan 609-735, Korea \\ ${ }^{2}$ Nanobiotechnology Center, Pusan National University, Pusan 609-735, Korea \\ ${ }^{3}$ The Division of Natural Medical Sciences, College of Health Science, Chosun University, Gwangju 501-759, Korea \\ *Present address: DNA Identification Center, National Forensic Service, Seoul 158-707, Korea \\ Correspondence to: Ho Sung Kang, e-mail: hspkang@pusan.ac.kr \\ Keywords: DIX-2, GLS1, Snail, epithelial-mesenchymal transition, glycolytic switch \\ Received: May 23, $2015 \quad$ Accepted: January 02, $2016 \quad$ Published: January 11, 2016
}

\section{ABSTRACT}

Most cancer cells depend on enhanced glucose and glutamine (GIn) metabolism for growth and survival. Oncogenic metabolism provides biosynthetic precursors for nucleotides, lipids, and amino acids; however, its specific roles in tumor progression are largely unknown. We previously showed that distal-less homeobox-2 (DIx-2), a homeodomain transcription factor involved in embryonic and tumor development, induces glycolytic switch and epithelial-mesenchymal transition (EMT) by inducing Snail expression. Here we show that DIx-2 also induces the expression of the crucial GIn metabolism enzyme glutaminase (GLS1), which converts GIn to glutamate. TGF- $\beta$ and Wnt induced GLS1 expression in a Dlx-2-dependent manner. GLS1 shRNA (shGLS1) suppressed in vivo tumor metastasis and growth. Inhibition of GIn metabolism by shGLS1, GIn deprivation, and GIn metabolism inhibitors (DON, 968 and BPTES) prevented DIx-2-, TGF- $\beta-$, Wnt-, and Snail-induced EMT and glycolytic switch. Finally, shDIx-2 and GIn metabolism inhibition decreased Snail mRNA levels through p53-dependent upregulation of Snail-targeting microRNAs. These results demonstrate that the DIx-2/GLS1/GIn metabolism axis is an important regulator of TGF- $\beta$ /Wntinduced, Snail-dependent EMT, metastasis, and glycolytic switch.

\section{INTRODUCTION}

Metabolic changes in cancer cells support higher rates of cell proliferation and growth compared to normal cells [1-8]. Most cancer cells rely on glycolysis more than mitochondrial oxidative phosphorylation for ATP production, even in the presence of oxygen; as a consequence, they exhibit increased glucose (Glc) uptake and lactate (Lac) production [1-3]. This phenomenon, termed the "Warburg effect" or glycolytic switch, is thought to increase the availability of biosynthetic precursors for nucleotides, lipids, and amino acids required for cancer cell proliferation. Glutamine (Gln) metabolism is also enhanced in many cancers $[1,2,4-8]$. Gln metabolism refills the pool of TCA cycle intermediates (anaplerosis), which are removed from mitochondria to participate in biosynthetic reactions for lipids, nucleic acids, and proteins (cataplerosis) [9-11]. Thus, Gln anaplerosis is crucial in maintaining cancer cell growth and development. Glutaminase (GLS), which converts Gln to glutamate, is the first enzyme in Gln anaplerosis $[10,11]$. There are two types of GLS in mammalian cells: the kidney type GLS1 and the liver type GLS2. GLS1 is important in cancer development and progression [12-17], and its levels are increased in many tumors, including breast and prostate cancer and hepatocellular carcinoma (HCC) tissues, compared to normal tissues [17, 18]. GLS1 shRNA (shGLS1), and the GLS-specific inhibitors bis-2(5-phenylacetamido-1,3,4-thiadiazol-2-yl)ethyl sulfide (BPTES) and 968, reduce the growth of several cancer types in xenograft models $[10,14,17]$.

Epithelial-mesenchymal transition (EMT) is essential for the initiation of metastasis, which is the most common cause of death in cancer patients [19-23]. EMT involves profound phenotypic changes, including the loss of epithelial cell polarity following reductions in levels 
of epithelial proteins, such as E-cadherin, and increases in levels of mesenchymal proteins, such as vimentin, that upregulate mesenchymal migration and invasion [19, 22]. EMT contributes to chemoresistance and cancer stem celllike phenotypes. Snail, the major transcription factor in EMT, triggers metastasis in response to several oncogenic signaling pathways, including transforming growth factor (TGF)- $\beta$ and Wnt, in breast carcinoma, prostate cancer, and colorectal cancer [24-28]. Recently, Snail has been shown to induce glycolytic switch, suppress mitochondrial respiration and cytochrome c oxidase activity [29], and suppress fructose-1,6-biphosphatase expression [30]. Glc metabolism may therefore regulate the induction of EMT.

Recently, we showed that distal-less homeobox-2 (Dlx-2), a homeodomain transcription factor involved in embryonic [31, 32] and tumor development [33-36], induces EMT and a glycolytic switch by increasing Snail expression [37]. Because Dlx-2 induces glycolytic switch via Snail induction, we postulated that Dlx-2 may activate other oncogenic metabolic pathways. We examined the effects of Dlx-2 on the expression of GLS1 and Gln metabolism. We also studied the impact of GLS1 knockdown on in vivo tumor metastasis and growth. To determine whether GLS1 and Gln metabolism influence TGF- $\beta$ /Wnt/Dlx-2/Snail-induced EMT and glycolytic switch, we examined p53-dependent regulation of Snailtargeting microRNAs (miRNAs) and Snail mRNA stability. Finally, we measured levels of Dlx-2, GLS1, Snail and Snail-targeting miRNAs in human cancer tissues. These experiments clarified the role of the Dlx-2/ GLS1 axis in TGF- $\beta$ /Wnt-induced, Snail-dependent EMT, metastasis, and glycolytic switch.

\section{RESULTS}

\section{GLS1 is induced by Dlx-2}

Because Dlx-2 induces glycolytic switch by inducing Snail expression [37], we postulated that Dlx-2 may activate other oncogenic metabolic pathways.

MCF-7 cells are non-invasive luminal A subtype breast cancer cells [38]. Dlx-2 and Snail induce EMT and glycolytic switch in MCF-7 cells [29, 30, 37]. Dlx-2 mRNA levels were lower in MCF-7 cells than in HCT116, HepG2, and HeLa cells; MCF-7, MDA-MB231, and A549 cells had similar Dlx-2 mRNA levels. Under normal conditions, Dlx-2 mRNA levels in the different cell lines were as follows relative to levels in HCT116 cells, which were used as a reference cell line: 0.446-fold in MCF-7, 0.351-fold in MDA-MB231, 0.322-fold in A549, 1.389fold in HepG2, and 1.144-fold in HeLa.

We examined potential metabolism-linked target genes of Dlx-2 using cDNA microarray technology (Agilent Human Genome 8x60K array, Agilent technologies, CA) and MCF-7 cells. Among 42,400 genes examined on this chip, Dlx-2 upregulated several metabolic enzymes, including GLS1, PFKFB2, H6PD, and ACACB. These enzymes are involved in Gln metabolism, glycolysis, pentose phosphate pathway (PPP), and fatty $\mathrm{acid} /$ cholesterol synthesis, respectively, suggesting that Dlx-2 may activate several oncogenic metabolic pathways (the microarray dataset is available in GSE61009). Dlx-2 led to a 2-fold upregulation of GLS1 (Figure 1A), without affecting the expression of other Gln metabolism enzymes, including GLS2, GLUD1, GOT1/2, and ME1 (data not shown).

Most cancer cells depend on enhanced Gln metabolism for growth and survival in addition to Glc metabolism [2, 4-8]. GLS1, which converts Gln to glutamate $[10,11]$, is the first enzyme involved in Gln anaplerosis. Because of its importance for Gln anaplerosis, we focused on Dlx-2-induced changes in GLS1 mRNA levels, even though they only increased 2-fold in the microarray data.

Real-time quantitative reverse transcription PCR (real-time qrtPCR, Figure 1B) and immunoblotting (Figure 1C) confirmed the microarray data; Dlx-2 overexpression increased GLS1 mRNA and protein levels. Because Snail acts downstream of Dlx-2, we examined the effects of shSnail on Dlx-2-induced GLS1 expression. shSnail did not inhibit Dlx-2-induced GLS1 expression (Figure 1D), suggesting that Dlx-2 induces GLS1 expression independently of Snail. In addition, Snail overexpression had no effect on GLS1 expression (data not shown).

We further examined the effects of Dlx-2 levels on GLS1 expression using a ChIP assay. Dlx-2 homeodomain binds DNA elements containing a TAAT core motif [31, 32]. Four putative Dlx-2 binding sites were found in the GLS1 promoter (Figure 1E). The ChIP assay showed that Dlx-2 binds to the GLS1 promoter (Figure 1E), suggesting that Dlx-2 may directly induce GLS1 expression.

Because Dlx-2 is induced by TGF- $\beta$ and Wnt [34, 37], we further investigated whether TGF- $\beta$ and Wnt3a induced GLS1 expression. TGF- $\beta$ and Wnt3a induced GLS1 expression (Figure 1F-1O). shDlx-2, but not shSnail, decreased TGF- $\beta$ - and Wnt3a-induced GLS1 expression (Figure 1F, 1G, $1 \mathrm{~K}$ and $1 \mathrm{~L}$ ), indicating that TGF- $\beta$ and Wnt3a induce GLS1 expression in a Dlx-2 dependent and Snail-independent manner. A ChIP assay showed that TGF- $\beta$ and Wnt3a increased Dlx-2 binding at the GLS1 promoter (Figure $1 \mathrm{H}$ and $1 \mathrm{M}$ ). TGF- $\beta$-induced GLS1 expression was also prevented by knockdown of Smad components of the TGF- $\beta$ signaling pathway (Figure 1I and 1J). sh $\beta$-catenin, shTCF4, and shAxin $1 / 2$ also suppressed Wnt3a-induced GLS1 expression (Figure $1 \mathrm{~N}$ and $1 \mathrm{O}$ ).

\section{shGLS1 inhibits in vivo tumor growth and metastasis}

Because shGLS1 and GLS inhibitors reduce growth in several types of cancer cell xenografts $[10,14,17]$, we 
examined the in vivo effects of shGLS1 on tumor growth and metastasis. HCT116 cells stably transfected with shCon or shGLS1 were injected subcutaneously into the dorsal flank of nude mice, which were then monitored for tumor growth. shGLS1 reduced tumor growth compared to shCon throughout the experiment (Figure 2A-2D). 28 days after injection, shGLS1 decreased tumor volume by $30 \%$ and weight by $64 \%$ relative to shCon (Figure $2 \mathrm{~A}-2 \mathrm{C}$ ).

Metastasis is the most common cause of death in cancer patients $[20,21,23]$. Thus, we investigated the effect of shGLS1 on pulmonary metastasis in vivo. HCT116 cells stably transfected with shCon or shGLS1 were injected into the lateral tail vein of nude mice. 48 days after injection, lungs were harvested to evaluate tumor metastasis. shGLS1 reduced the number of metastatic nodules (Figure 2E-2G) and the number of micrometastatic lesions (Figure 2F). While shCon cell injections resulted in an average of 55 metastatic nodules per lung, shGLS1 cell injections resulted in an average of 10 nodules per lung (Figure $2 \mathrm{G}$ ). These results show that GLS1 levels are important in in vivo tumor metastasis as well as tumor growth.

\section{Inhibition of GIn metabolism prevents EMT}

EMT is closely associated with tumor metastasis [19-22]. Thus, we examined the effects of GLS1 knockdown on EMT in MCF-7 cells. shGLS1 prevented Dlx-2-induced EMT and E-cadherin downregulation (Figure 3A-3C). In addition, shGLS1 prevented TGF- $\beta$ and Wnt3a-induced EMT and E-cadherin downregulation (Figure 3D-3I). In contrast, shGLS2 did not inhibit Dlx-2-, TGF- $\beta$-, or Wnt3a-induced EMT (data not shown).

We then examined the effects of shGLS1 in Snail overexpressing cells. We used 2 different GLS1
A

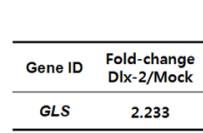

B

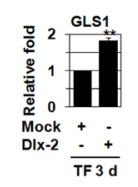

C

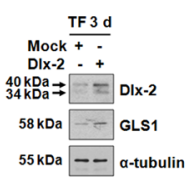

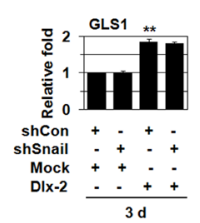

$\mathbf{E}$

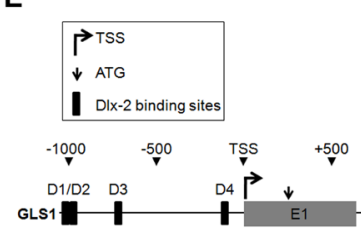

I

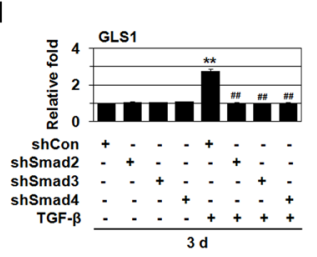

$\mathrm{N}$

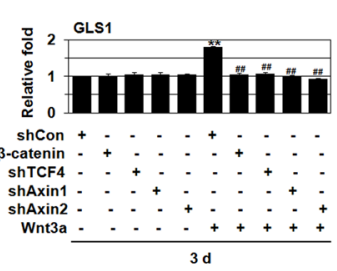

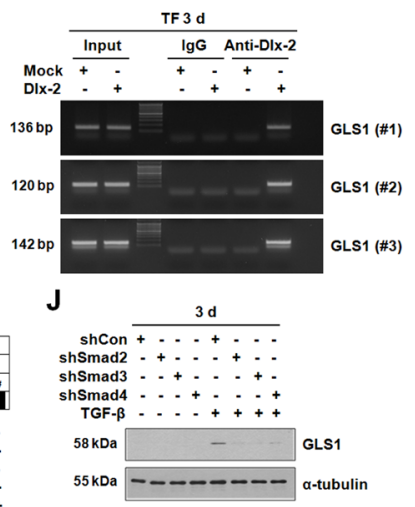

0

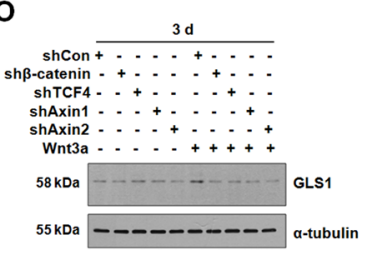

Figure 1: TGF- $\boldsymbol{\beta}$ and Wnt3a induces GLS1 expression by Dlx-2 activation. A-C. MCF-7 cells were transfected with Dlx-2. Changes in cellular gene transcription were detected by microarray analysis (A). Fold increases in expression as compared with Mock are shown. The cells were also analyzed by real-time qrtPCR (B) and immunoblotting (C) using the indicated primers and antibodies. $* * p<$ 0.01 versus Mock. D. MCF-7 cells co-transfected with Dlx-2 and shSnail were analyzed by real-time qrtPCR for GLS1 expression. ** $p<$ 0.01 versus Mock. E. A schematic diagram of the human GLS1 promoter regions is shown in left panel, and the 4 predicted Dlx-2 binding sites are indicated by black boxes and numbered D1, D2, D3 and D4. The ChIP-enriched DNA was amplified using primers \#1, \#2 or \#3, which encompass the D1/D2, D3, or D4 binding sites in the GLS1 promoter, respectively. MCF-7 cells were transfected with Dlx-2 and analyzed using ChIP assays (right panel). F, G. MCF-7 cells transfected with shDlx-2 or shSnail and then treated with TGF- $\beta$ were analyzed by real-time qrtPCR (F) and immunoblotting (G) for GLS1 expression. ${ }^{*} p<0.01$ versus untreated, ${ }^{*} p<0.01$ versus shCon. H. MCF-7 cells were treated with TGF- $\beta$ and analyzed using ChIP assays. I, J. MCF-7 cells were transfected with shRNA for Smad 2/3/4 and then treated with TGF- $\beta$. The cells were then analyzed by real-time qrtPCR (I) and immunoblotting (J) for GLS1 expression. ** $p<0.01$ versus untreated, ${ }^{\#} p<0.01$ versus shCon. K, L. MCF-7 cells transfected with shDlx-2 or shSnail and then treated with Wnt3a CM were analyzed by real-time qrtPCR (K) and immunoblotting (L) for GLS1 expression. ${ }^{* *} p<0.01$ versus untreated, ${ }^{\# \#} p<0.01$ versus shCon. M. MCF-7 cells were treated with Wnt3a CM and analyzed using ChIP assays. N, O. MCF-7 cells were transfected with shRNA for $\beta$-catenin, TCF4, and Axin $1 / 2$ and then treated with Wnt3a CM. The cells were then analyzed by real-time qrtPCR (N) and immunoblotting (O) for GLS1 expression. ${ }^{* *} p<0.01$ versus untreated, ${ }^{\# \#} p<0.01$ versus shCon. All error bars represent SE. For all immunoblotting images, cropped blots are shown. 
shRNAs (shGLS1 \#1 and \#2), both of which effectively reduced GLS1 levels. Both shGLS1s prevented Snailinduced EMT and E-cadherin downregulation in the Snail-overexpressing MCF-7 cells (Figure 3J-3L). Thus, although GLS1 levels were not changed by Snail overexpression, Snail-induced EMT was inhibited by shGLS1.

We also examined the effects of Gln deprivation on EMT. Gln deprivation suppressed Dlx-2-induced EMT, E-cadherin downregulation, and vimentin upregulation (Supplemental Figure 1A-C). Additionally, Gln deprivation suppressed TGF- $\beta$-induced EMT and E-cadherin downregulation (Supplemental Figure 1D and 1E). Gln deprivation also suppressed Snailinduced EMT, E-cadherin downregulation, and vimentin upregulation (Supplemental Figure 1F-H). In addition, the Gln analog 6-diazo-5-oxo-L-norleucine (DON) and two GLS-selective inhibitors, compound 968 and BPTES [16], suppressed TGF- $\beta$-induced EMT and E-cadherin downregulation (Supplemental Figure 1I-N).

A

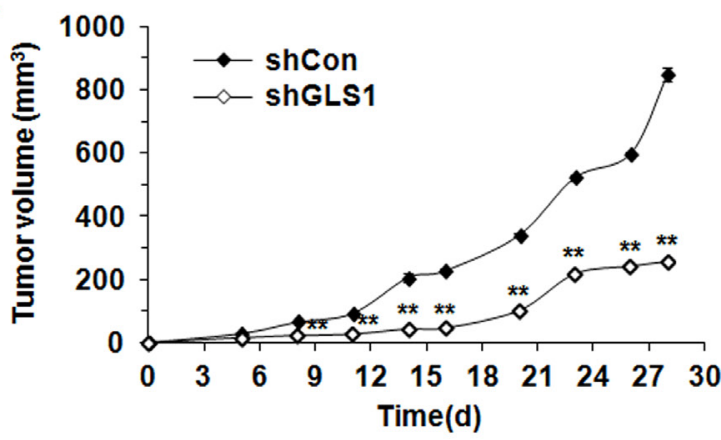

B
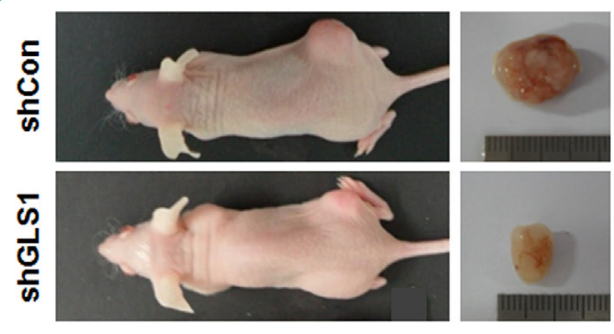

C
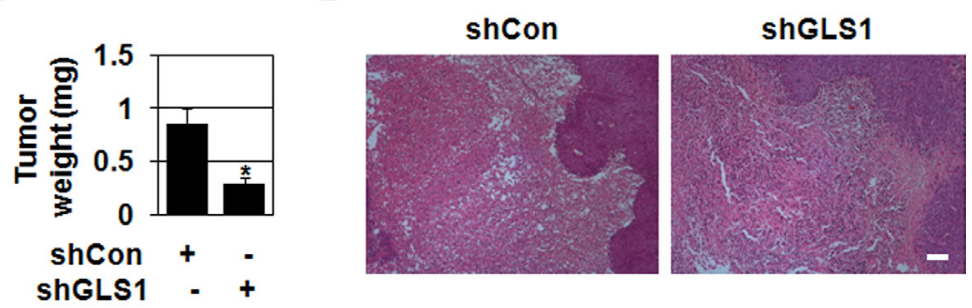

E
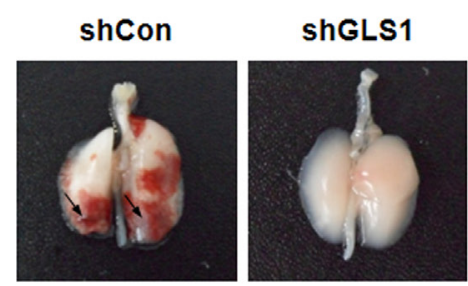

F
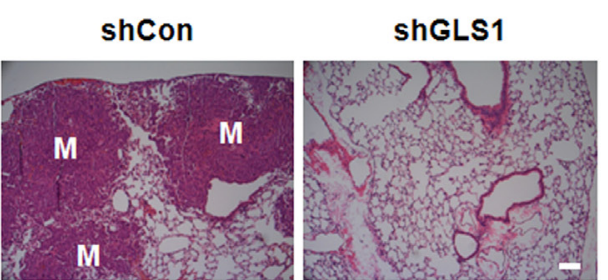

G

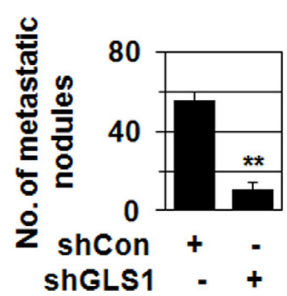

Figure 2: shGLS1 inhibits tumor growth and metastasis. A-D. HCT116 cells stably transfected with shCon or shGLS1 were injected subcutaneously into the dorsal flank of nude mice $(n=4-5)$. Tumor growth curves (A) and photographs of representative mice, tumors (B), tumor weight (C) and H\&E staining (D) are shown. ${ }^{*} p<0.05$; $* * p<0.01$ versus shCon. E-G. $1 \times 10^{6}$ HCT116 cells stably transfected with shCon or shGLS1 were injected into the tail vein of nude mice $(n=3-5)$. Photographs of representative lungs (E) and H\&E staining of lung sections are shown (F). The number of metastatic nodules $(\mathrm{G}){ }^{* *} p<0.01$ versus shCon. All error bars represent SE. All scale bars represent $100 \mu \mathrm{m}$. 
metabolism reduced EMT not only in MCF-7 cells, but also in HCT116 and MDCK cells.

\section{GIn metabolism inhibition and shDlx-2 decrease Snail mRNA levels}

Because Gln metabolism inhibition prevents Dlx-2-, TGF- $\beta-$, Wnt3a-, and Snail-induced EMT, we examined the effects of Gln metabolism on Snail expression.
shGLS1 or Gln deprivation reduced Snail expression in Dlx-2-overexpressing cells (Figure 3B and Supplemental Figure 1B). In addition, Gln metabolism inhibition via shGLS1, Gln deprivation, DON, 968, or BPTES also prevented TGF- $\beta$-induced Snail expression (Figure 3E and $3 \mathrm{~F}$, and Supplemental Figure 1E, $1 \mathrm{~J}, 1 \mathrm{~L}$, and $1 \mathrm{~N}$ ). $\alpha-K G$ prevented the reduction of TGF- $\beta$-induced Snail expression following Gln metabolism inhibition (Gln deprivation or DON) (Supplemental Figure 1E and 1J).
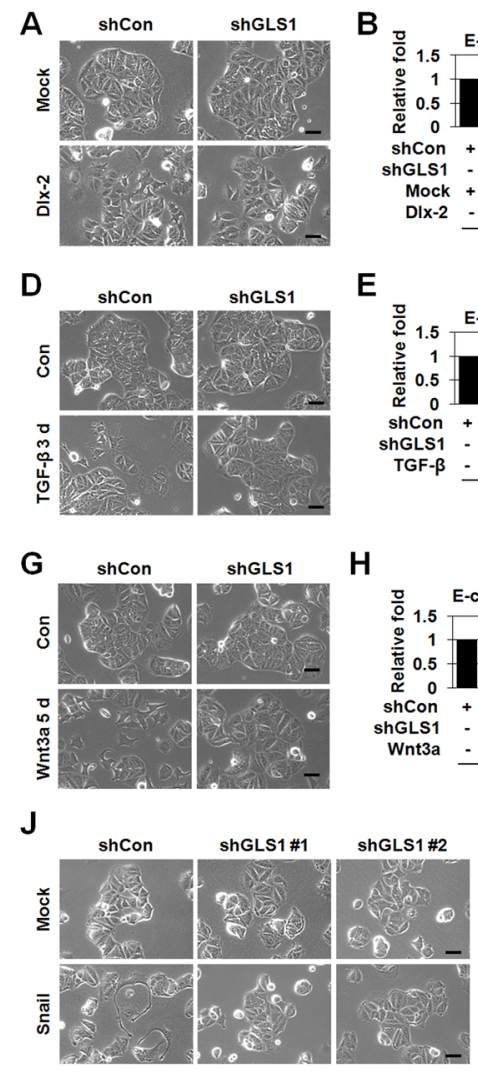

M

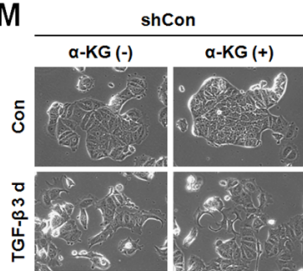

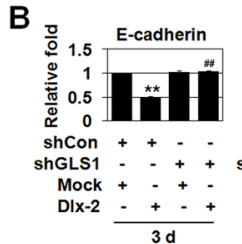

E

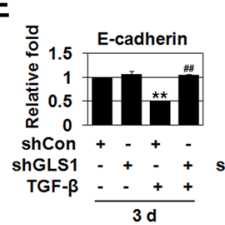

H

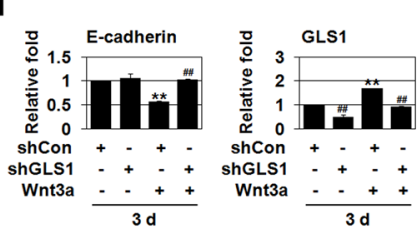

K

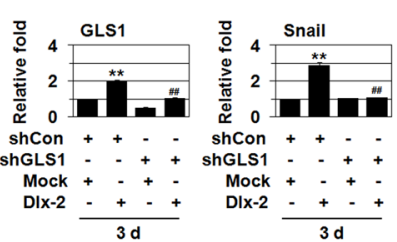

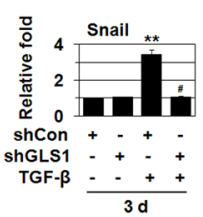

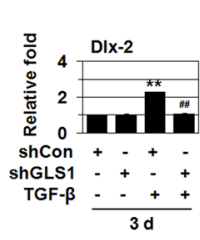

I

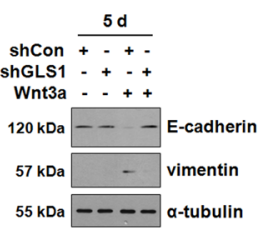

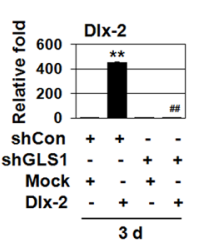

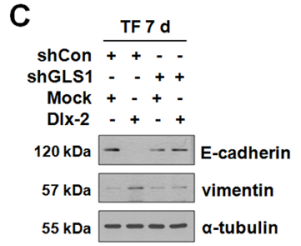

$\mathbf{F}$

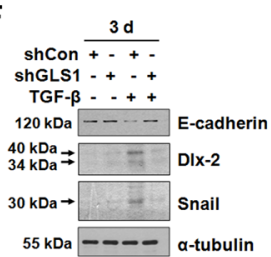

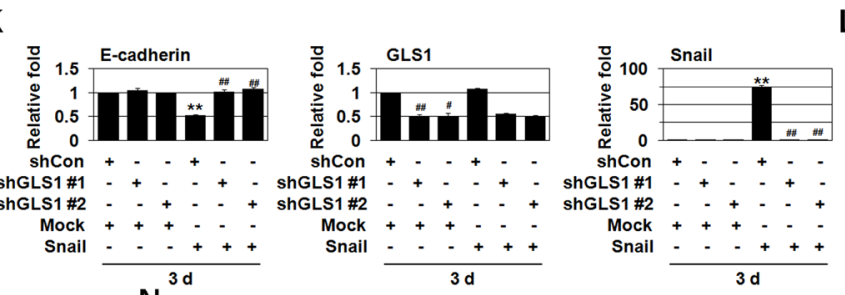

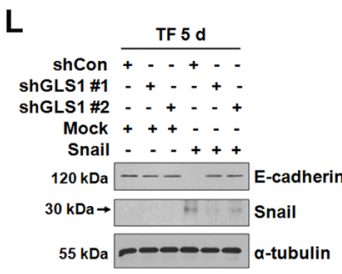

N
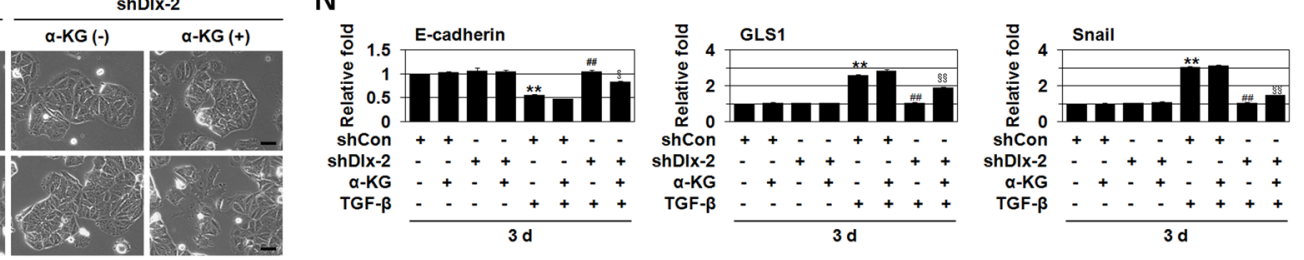

Figure 3: Inhibition of Gln metabolism prevents EMT. A-C. MCF-7 cells were co-transfected with Dlx-2 and shGLS1. The cells were analyzed for cell morphology by phase-contrast microscopy (A). The cells were also analyzed by real-time qrtPCR (B) and immunoblotting (C) using the indicated primers and antibodies. ${ }^{* *} p<0.01$ versus Mock, ${ }^{\#} p<0.01$ versus shCon. D-I. MCF-7 cells were transfected with shGLS1 and then treated with TGF- $\beta$ (D-F) or Wnt3a CM (G-I). The cells were analyzed by phase-contrast microscopy for cell morphology $(\mathrm{D}, \mathrm{G})$, and by real-time $\mathrm{qrtPCR}(\mathrm{E}, \mathrm{H})$ and immunoblotting $(\mathrm{F}, \mathrm{I})$ using the indicated primers and antibodies. $* * p<0.01$ versus untreated, ${ }^{\#} p<0.05 ;{ }^{\#} p<0.01$ versus shCon. J-L. MCF-7 cells were co-transfected with Snail and 2 different shGLS1 constructs (\#1 and \#2). The cells were analyzed for cell morphology by phase-contrast microscopy (J). The cells were also analyzed by real-time qrtPCR $(\mathrm{K})$ and immunoblotting (L) using the indicated primers and antibodies. ${ }^{* *} p<0.01$ versus Mock, ${ }^{\#} p<0.05$; ${ }^{*} p<0.01$ versus shCon. M, N. MCF-7 cells were transfected with shDlx-2 and then treated with TGF- $\beta$ in the presence or absence of $\alpha-K G$ and analyzed for cell morphology by phase-contrast microscopy (M). The cells were also analyzed by real-time qrtPCR for E-cadherin, GLS1, and Snail expression (N). ${ }^{* *} p<0.01$ versus untreated, ${ }^{\# \#} p<0.01$ versus shCon, ${ }^{\S} p<0.05 ;{ }^{\S} p<0.01$ versus shDlx-2 in the absence of $\alpha-\mathrm{KG}$. All error bars represent SE. All scale bars represent $100 \mu \mathrm{m}$. For all immunoblotting images, cropped blots are shown. 
shGLS1 or Gln deprivation also decreased Snail expression in Snail-overexpressing cells (Figure $3 \mathrm{~K}$ and 3L, and Supplemental Figure 1G). Dlx-2 expression was also reduced by Gln metabolism inhibition (shGLS1, Gln deprivation, or DON) in Dlx-2-overexpressing cells and TGF- $\beta$-treated cells (Figure 3B, 3E, and 3F, and Supplemental Figure 1B, 1E, and 1J). Furthermore, shDlx-2 suppressed the TGF- $\beta$-mediated increase in Snail mRNA levels; $\alpha-K G$ prevented the inhibitory effects of shDlx-2 on TGF- $\beta$-induced Snail mRNA expression (Figure 3N).

Gln metabolism inhibition had similar effects on Snail expression in HCT116 and MDCK cells. shGLS1 prevented Dlx-2- and Snail-induced Snail expression in HCT116 cells (Supplemental Figure 2B and 2F). shGLS1 also inhibited TGF- $\beta$ - and Snail-induced Snail expression in MDCK cells (Supplemental Figure 2H and 2J). Thus, Gln metabolism inhibition decreased Snail expression in HCT116 and MDCK cells in addition to MCF-7 cells.

Although co-transfection of Dlx-2 and Snail did not affect Snail expression, shDlx-2 and Snail co-transfection significantly reduced Snail levels (Supplemental Figure $3 \mathrm{~A}$ ), indicating that Dlx-2 is required for the maintenance of Snail mRNA levels. Gln metabolism inhibition and shDlx-2 together may therefore affect Snail mRNA stability. We examined this possibility by treating Snailtransfected cells with actinomycin D in the presence or absence of Gln. As expected, Snail mRNA stability was significantly reduced by Gln deprivation compared to normal growth medium (Supplemental Figure 3B). Snail mRNA had a half-life $\left(\mathrm{t}_{1 / 2}\right)$ of $9.99 \mathrm{~h}$ in complete medium and $\mathrm{a} \mathrm{t}_{1 / 2}$ of $3.57 \mathrm{~h}$ in Gln-free medium; the absence of Gln accelerated Snail mRNA decay. Thus, in addition to inducing Snail gene transcription, Dlx-2 upregulates Snail levels by stimulating Gln metabolism, which increases Snail mRNA stability.

\section{Gln metabolism inhibition and shDlx-2 increase the expression of Snail-targeting miRNAs through p53 regulation}

Snail mRNA levels are suppressed by many miRNAs, including miR-23b, miR-29b, miR-30, miR34, miR-125b, miR-148a, miR-153, miR-200, miR-203, miR let-7, miR-7, miR-9, miR-128-2, miR-145, and miR204 (39; Supplemental references 1-17). We examined whether Gln metabolism inhibition affects the expression of these Snail-targeting miRNAs. shGLS1, DON, and Gln deprivation increased the expression of miR-23b, miR29b, miR-30, miR-34, miR-125b, miR-148a, miR-153, miR-200, and miR-203 without affecting the expression of miR let-7, miR-7, miR-9, miR-128-2, miR-145, and miR-204 (Table 1). Similar results were obtained in Dlx-2 knockdown cells (Table 1), $\alpha-\mathrm{KG}$ inhibited the effects of shGLS1, Gln deprivation, and shDlx-2 on the expression of the Snail-targeting miRNAs (Table 1).
We also found that TGF- $\beta$ reduced the expression of the Snail-targeting miRNAs (Supplemental Figure 4A). These results suggest that TGF- $\beta$ upregulates Snail levels by reducing the expression of Snail-targeting miRNAs in addition to directly increasing Snail gene expression. Gln deprivation suppressed the inhibitory effects of TGF- $\beta$ on Snail-targeting miRNA levels (Supplemental Figure 4A). In addition, Dlx-2 overexpression reduced the expression of the Snail-targeting miRNAs (Supplemental Figure 4B). Thus, Dlx-2 increased Snail levels by repressing Snailtargeting miRNA expression; furthermore, Dlx-2 may downregulate Snail-targeting miRNAs through regulation of Gln metabolism.

Tumor cells exhibit enhanced Glc and Gln uptake and are especially sensitive to nutritional stress. The tumor suppressor p53, which reprograms tumor cell functions in response to nutritional stress, is a major sensor of low nutrient levels [40]. Therefore, we investigated whether p53 is involved in the expression of Snail-targeting miRNAs. Gln metabolism inhibition (shGLS1, DON, or Gln deprivation) and shDlx-2 increased p53 expression (Figure 4A-4D), while $\alpha-K G$ suppressed shGLS1- and shDlx-2 induced p53 expression (Figure 4A and 4B). TGF- $\beta$ decreases $\mathrm{p} 53$ by inhibiting its transcription and translation [41]. We found that shDlx-2 prevented the TGF- $\beta$-mediated decrease in p53 levels, but shSnail did not (Figure 4E and 4F). This indicates that TGF- $\beta$ decreases $\mathrm{p} 53$ via Dlx-2. The shDlx-2-mediated increase in p53 levels was also prevented by TGF- $\beta$ (Figure $4 \mathrm{E}$ and $4 \mathrm{~F}$ ).

In addition, shp53 suppressed the expression of all of 9 Snail-targeting miRNAs induced by Gln metabolism inhibition (shGLS1, DON, and Gln deprivation) and shDlx-2 (Table 1). Thus, p53 induces the expression of other miRNAs besides miR-34 and miR-200 during Gln metabolism inhibition.

We then examined the role of p53 in shDlx-2and Gln metabolism inhibition-mediated suppression of TGF- $\beta$-induced EMT. shp53 promoted TGF- $\beta$-induced EMT (Figure 4G and 4H). shp53 prevented shDlx-2- and shGLS1-mediated suppression of TGF- $\beta$-induced EMT (Figure 4G). shp53 prevented Gln deprivation-mediated suppression of TGF- $\beta$-induced EMT in MCF-7 cells as well (Figure 4H), suggesting that TGF- $\beta$ induces EMT in a p53-dependent manner. In summary, Dlx-2 and Gln metabolism may downregulate Snail-targeting miRNAs in a p53-dependent manner during TGF- $\beta$-induced EMT.

\section{Inhibition of Gln metabolism prevents Dlx-2/ TGF-ß/Wnt3a/Snail-induced glycolytic switch and mitochondrial repression}

Previously, we showed that Snail induces glycolytic switch and mitochondrial repression [29]. Dlx-2 induced glycolytic switch and mitochondrial repression (Figure 5A and Supplemental Figure 5A). Next, we examined whether 
Table 1: p53-dependent regulation of Snail-targeting miRNAs by GIn metabolism inhibition and shDlx-2

\begin{tabular}{|c|c|c|c|c|c|c|c|c|c|c|c|c|c|c|c|c|c|}
\hline \multirow[t]{2}{*}{ Genes } & \multirow{2}{*}{$\frac{\text { shGLS1 }}{3 \mathrm{~d}}$} & \multicolumn{2}{|c|}{ shGLS1 } & \multicolumn{2}{|c|}{ shGLS1 } & \multicolumn{2}{|c|}{ DON } & \multirow{2}{*}{$\frac{\operatorname{Gln}(-)}{3 \mathrm{~d}}$} & \multicolumn{2}{|c|}{ Gln (-) } & \multicolumn{2}{|c|}{ GIn (-) } & \multirow{2}{*}{$\frac{\operatorname{shDlx}-2}{3 \mathrm{~d}}$} & \multicolumn{2}{|c|}{ shDlx-2 } & \multicolumn{2}{|c|}{ shDlx-2 } \\
\hline & & $\begin{array}{c}\alpha-\mathbf{K G} \\
(-)\end{array}$ & $\begin{array}{c}\alpha-\mathbf{K G} \\
(+)\end{array}$ & shCon & $\operatorname{shp53}$ & shCon & shp53 & & $\begin{array}{c}\alpha-K G \\
(-)\end{array}$ & $\begin{array}{c}\alpha-\mathbf{K G} \\
(+)\end{array}$ & shCon & shp53 & & $\begin{array}{c}\alpha-\mathbf{K G} \\
(-)\end{array}$ & $\begin{array}{c}\alpha-\mathbf{K G} \\
(+)\end{array}$ & shCon & shp53 \\
\hline miR-23b & $2.813^{\text {aa }}$ & $2.886^{\text {aa }}$ & $1.095^{\mathrm{bb}}$ & $2.923^{\text {aa }}$ & $1.084^{\mathrm{bb}}$ & $2.464^{\text {aa }}$ & $1.068^{\mathrm{bb}}$ & $2.525^{\mathrm{aa}}$ & $2.491^{\text {aa }}$ & $0.996^{\mathrm{b}}$ & $2.508^{\mathrm{aa}}$ & $1.093^{\mathrm{bb}}$ & $1.871^{\mathrm{aa}}$ & $1.872^{\mathrm{aa}}$ & $1.013^{\mathrm{bb}}$ & $1.890^{\mathrm{aa}}$ & $1.088^{\mathrm{bb}}$ \\
\hline miR-29b & $1.551^{\text {aa }}$ & $1.576^{\mathrm{aa}}$ & $1.046^{\mathrm{b}}$ & $1.571^{\text {aa }}$ & $1.054^{\mathrm{bb}}$ & $2.037^{\mathrm{a}}$ & $1.055^{\mathrm{b}}$ & $2.021^{\text {aa }}$ & $2.071^{\text {aa }}$ & $0.997^{\mathrm{b}}$ & $1.828^{\mathrm{a}}$ & $1.046^{\mathrm{b}}$ & $1.562^{\mathrm{aa}}$ & $1.610^{\mathrm{aa}}$ & $0.981^{\mathrm{bb}}$ & $1.541^{\mathrm{aa}}$ & $1.040^{\mathrm{bb}}$ \\
\hline miR-30 & $2.505^{\mathrm{aa}}$ & $2.532^{\mathrm{aa}}$ & $0.877^{\text {bb }}$ & $2.548^{\text {aa }}$ & $1.018^{\mathrm{bb}}$ & $2.382^{\mathrm{a}}$ & $1.127^{\mathrm{b}}$ & $2.465^{\mathrm{aa}}$ & $2.493^{\text {aa }}$ & $0.954^{\mathrm{bb}}$ & $2.414^{\mathrm{aa}}$ & $1.058^{\mathrm{bb}}$ & $1.848^{\text {aa }}$ & $1.823^{\mathrm{aa}}$ & $1.003^{\mathrm{b}}$ & $1.859^{\text {aa }}$ & $1.063^{\mathrm{bb}}$ \\
\hline miR-34 & $2.156^{\mathrm{aa}}$ & $2.135^{\text {aа }}$ & $1.004^{\mathrm{bb}}$ & $2.175^{\text {aa }}$ & $1.020^{\mathrm{bb}}$ & $2.623^{\mathrm{a}}$ & $1.071^{\mathrm{b}}$ & $2.464^{\text {aa }}$ & $2.448^{\text {aa }}$ & $0.937^{\mathrm{b}}$ & $2.365^{\mathrm{aa}}$ & $1.039^{\mathrm{bb}}$ & $1.951^{\text {aa }}$ & $1.835^{\mathrm{a}}$ & $0.914^{\mathrm{bb}}$ & $1.918^{\text {aa }}$ & $1.003^{\mathrm{bb}}$ \\
\hline miR-125b & $3.544^{\mathrm{aa}}$ & $3.570^{\mathrm{a}}$ & $1.024^{\mathrm{b}}$ & $3.656^{\mathrm{aa}}$ & $1.081^{\mathrm{bb}}$ & $3.185^{\mathrm{a}}$ & $1.045^{\mathrm{b}}$ & $3.215^{\mathrm{aa}}$ & $3.078^{\mathrm{aa}}$ & $1.051^{\mathrm{bb}}$ & $3.324^{\text {aa }}$ & $1.053^{\mathrm{bb}}$ & $2.983^{\text {aа }}$ & $3.045^{\mathrm{aa}}$ & $1.052^{\mathrm{bb}}$ & $2.880^{\text {aa }}$ & $1.101^{\mathrm{bb}}$ \\
\hline miR-148a & $3.139^{\mathrm{aa}}$ & $3.267^{\text {aa }}$ & $0.917^{\mathrm{b}}$ & $3.048^{\mathrm{aa}}$ & $1.043^{\mathrm{bb}}$ & $2.874^{\mathrm{a}}$ & $1.073^{\mathrm{b}}$ & $3.003^{\text {aa }}$ & $3.052^{\mathrm{aa}}$ & $1.023^{\mathrm{bb}}$ & $2.919^{\text {aa }}$ & $1.062^{\mathrm{bb}}$ & $2.356^{\mathrm{aa}}$ & $2.438^{\mathrm{aa}}$ & $1.018^{\mathrm{bb}}$ & $2.239^{\mathrm{aa}}$ & $1.007^{\mathrm{bb}}$ \\
\hline miR-153 & $3.124^{\mathrm{aa}}$ & $3.130^{\text {aa }}$ & $1.150^{\mathrm{bb}}$ & $3.218^{\text {aa }}$ & $1.080^{\mathrm{bb}}$ & $2.458^{\mathrm{a}}$ & $1.092^{\mathrm{b}}$ & $2.577^{\text {aа }}$ & $2.501^{\text {aa }}$ & $1.178^{\mathrm{b}}$ & $2.770^{\text {aa }}$ & $1.108^{\mathrm{bb}}$ & $2.472^{\mathrm{aa}}$ & $2.599^{\mathrm{aa}}$ & $0.981^{\mathrm{bb}}$ & $2.344^{\text {aa }}$ & $1.043^{\mathrm{bb}}$ \\
\hline miR-200 & $2.616^{\mathrm{aa}}$ & $2.700^{\text {aa }}$ & $0.930^{\mathrm{bb}}$ & $2.618^{\text {aa }}$ & $1.067^{\mathrm{bb}}$ & $2.261^{\mathrm{a}}$ & $1.070^{\mathrm{b}}$ & $2.472^{\text {aa }}$ & $2.471^{\mathrm{aa}}$ & $1.032^{\mathrm{bb}}$ & $2.480^{\mathrm{aa}}$ & $1.082^{\mathrm{bb}}$ & $2.183^{\text {aa }}$ & $2.318^{\text {aa }}$ & $0.993^{\text {bb }}$ & $2.123^{\text {aa }}$ & $1.074^{\mathrm{bb}}$ \\
\hline miR-203 & $2.257^{\text {aa }}$ & $2.363^{\text {aa }}$ & $1.167^{\mathrm{bb}}$ & $2.289^{\mathrm{aa}}$ & $1.073^{\mathrm{bb}}$ & $2.196^{\mathrm{a}}$ & $1.083^{\mathrm{b}}$ & $2.376^{\mathrm{aa}}$ & $2.312^{\mathrm{aa}}$ & $1.078^{\mathrm{bb}}$ & $2.380^{\mathrm{aa}}$ & $1.037^{\mathrm{bb}}$ & $2.271^{\mathrm{aa}}$ & $2.306^{\mathrm{aa}}$ & $1.038^{\mathrm{bb}}$ & $2.223^{\mathrm{aa}}$ & $1.044^{\mathrm{bb}}$ \\
\hline miR let-7 & 1.001 & & & & & & & 1.075 & 1.016 & 1.107 & & & 0.964 & & & & \\
\hline miR-7 & 1.230 & & & & & & & 1.264 & 1.306 & 1.386 & & & 1.206 & & & & \\
\hline miR-9 & 0.917 & & & & & & & 0.899 & 0.846 & 0.866 & & & 1.064 & & & & \\
\hline miR-128-2 & 1.275 & & & & & & & 1.119 & 1.022 & 0.981 & & & 1.061 & & & & \\
\hline miR-145 & 0.763 & & & & & & & 1.051 & 1.106 & 0.929 & & & 1.066 & & & & \\
\hline miR-204 & 1.102 & & & & & & & 1.123 & 1.069 & 1.103 & & & 1.020 & & & & \\
\hline
\end{tabular}

GLS1, glutaminase; DON, 6-diazo-5-oxo-L-norleucine; Gln, glutamine; Dlx-2, distal-less (Dlx) homeobox 2; $\alpha$-KG, dimethyl- $\alpha$-ketoglutarate; miR, microRNA.

MCF-7 cells were transfected with shGLS1, cultured in complete or Gln-free medium or transfected with shDlx-2, and then treated with $\alpha-K G$ or co-transfected with shp53. MCF-7 cells were transfected with shp53 and then treated with DON; The cells were analyzed by real-time qrtPCR.

${ }^{\mathrm{a}} p<0.05 ;{ }^{\text {aa }} p<0.01$ versus control (untreated and shCon).

${ }^{\mathrm{b}} p<0.05 ;{ }^{\mathrm{bb}} p<0.01$ versus shGLS1, DON, Gln-free medium, or shDlx-2.

Gln metabolism affects Dlx-2-induced glycolytic switch and mitochondrial repression. shGLS1 and Gln deprivation suppressed Dlx-2-induced Glc consumption and Lac production (Figure 5A and Supplemental Figure 5A). In addition, shGLS1 and Gln deprivation prevented Dlx-2induced repression of oxygen $\left(\mathrm{O}_{2}\right)$ consumption (Figure 5A and Supplemental Figure 5A). ATP levels in these cells were similar to those in control cells (data not shown). By measuring $\mathrm{O}_{2}$ consumption and Lac production, we estimated the relative contributions of glycolysis and aerobic respiration to total ATP production. shGLS1 and Gln deprivation prevented the Dlx-2-mediated increase in the ratio of ATP produced by glycolysis versus aerobic respiration (Figure 5A and Supplemental Figure 5A). This indicates that Gln metabolism inhibition suppresses Dlx2-induced glycolytic switch and mitochondrial repression.

We then examined whether Gln metabolism is linked to TGF- $\beta /$ Wnt-induced glycolytic switch and mitochondrial repression. TGF- $\beta$ and Wnt induced glycolytic switch and mitochondrial repression (Figure $5 \mathrm{~B}$ and $5 \mathrm{C}$ ). shGLS1 suppressed TGF- $\beta$ - and Wnt-induced Glc consumption and Lac production (Figure 5B and 5C). In addition, shGLS1 prevented TGF- $\beta$ - and Wnt-induced repression of $\mathrm{O}_{2}$ consumption (Figure 5B and 5C). Total ATP concentrations remained the same in all cells (data not shown). shGLS1 inhibited the TGF- $\beta$ - and Wnt-mediated increase in the ratio of ATP produced by glycolysis versus aerobic respiration (Figure 5B and 5C). This indicates that shGLS1 suppresses TGF- $\beta$ /Wnt-induced glycolytic switch and mitochondrial repression. Thus, Gln metabolism appears to be linked to Dlx-2-, TGF- $\beta$-, and Wnt-induced glycolytic switch and mitochondrial repression.

Finally, we examined whether Gln metabolism affects Snail-induced glycolytic switch and mitochondrial repression (Figure 5D and Supplemental Figure 5B). Similar to their inhibitory effects on EMT, shGLS1 and Gln deprivation prevented Snail-induced glycolytic switch in Snail-overexpressing cells (Figure 5D and Supplemental Figure 5B). shGLS1 and Gln deprivation also prevented Snail-induced repression of $\mathrm{O}_{2}$ consumption (Figure 5D and Supplemental Figure 5B). Thus, Gln metabolism inhibition suppressed Snail-induced glycolytic switch and mitochondrial repression.

\section{Expression of Dlx-2, GLS1, Snail, p53, and Snail-targeting miRNAs in human tumors}

To examine the physiological relevance of the Dlx2/GLS1/p53/miRNA/Snail cascade, we analyzed their levels in human tumor samples. Dlx-2, GLS1, Snail, p53, 
and Snail-targeting miRNA expression was examined with real-time qrtPCR using RNAs extracted from paired biopsies of breast, colon, and ovarian cancer and corresponding normal tissues. Dlx-2 and Snail expression were higher in breast cancer tissues compared to matched non-tumorigenic tissues (Figure 6A). GLS1 expression was also higher, and p53 expression was lower, in breast cancer tissues (Figure 6A). In addition, Dlx-2, GLS1, and Snail expression were higher, and p53 expression was lower, in colon and ovarian cancer tissues compared to matched normal tissues regardless of cancer stage (Figure 6B-6D). The expression of most Snail-targeting miRNAs (miR-23b, miR-29b, miR-30, miR-125b, miR153, and miR-200) was lower in breast and colon cancer than in matched normal tissues; however, miR-34, miR148a, and miR-203 expression was similar to control tissues in metaplastic breast carcinoma (Figure 6E). We also examined the expression of Dlx-2, GLS1, and
A

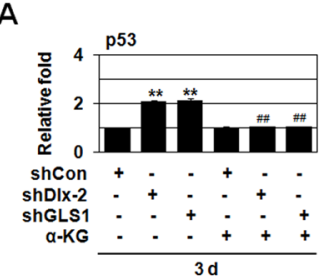

E

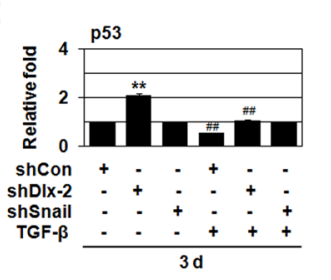

G

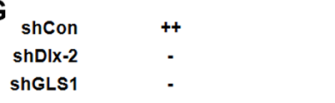

ShGLS1
shp53
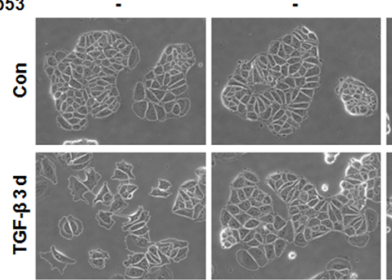

H

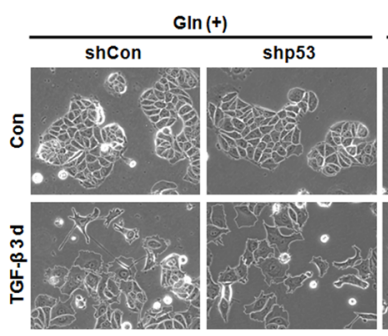

B
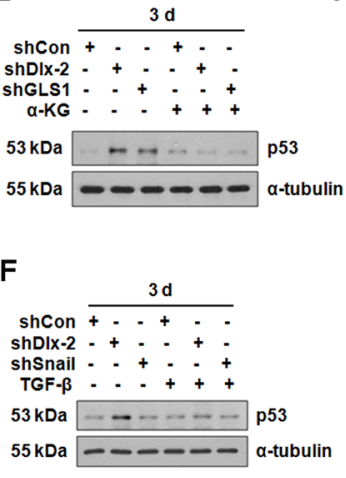

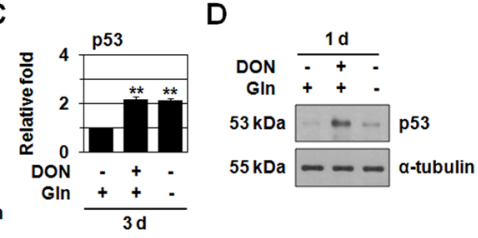<smiles>C1CCCCC1</smiles>

$$
\text { . }
$$


Snail protein using immunoblotting. The expression of all three was higher in breast, colon and ovarian cancer tissues than in matched non-tumorigenic tissues (Figure 6A-6D). These results support important roles for Dlx-2, GLS1, and p53 in tumor development.

\section{DISCUSSION}

Dlx-2, a transcriptional factor involved in embryonic development, tissue homeostasis, and the cell cycle [31, $32]$, is important in carcinogenesis; Dlx-2 expression
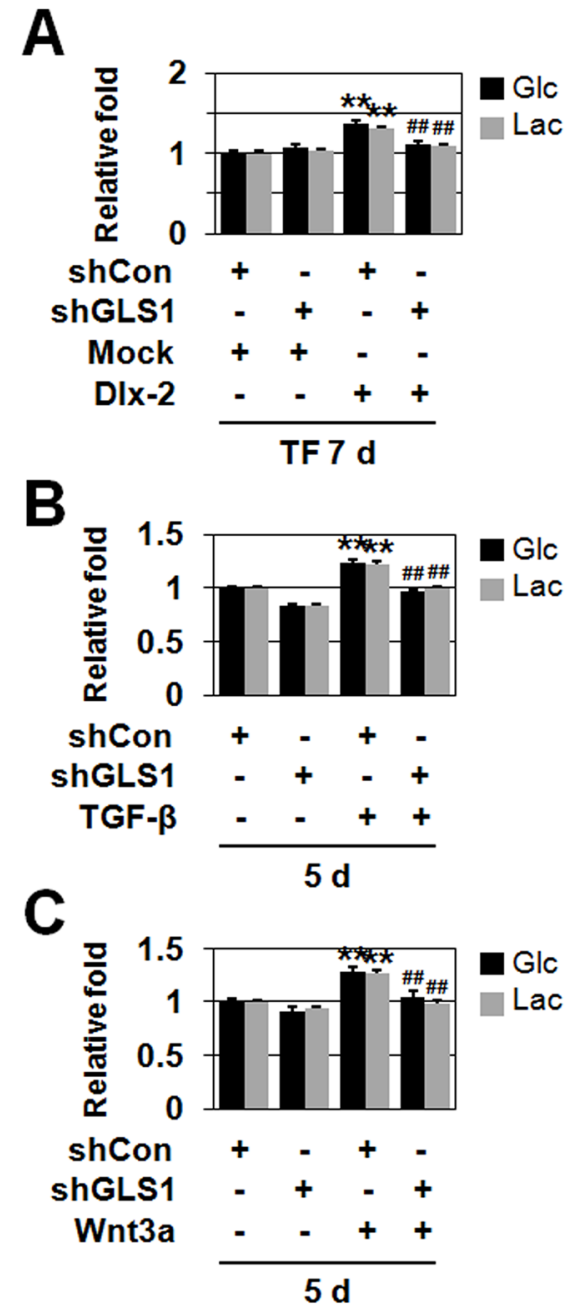

D

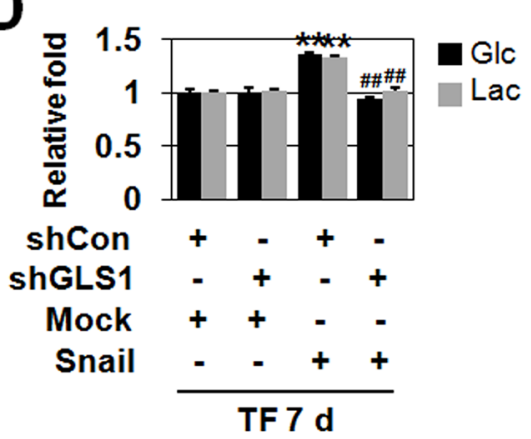

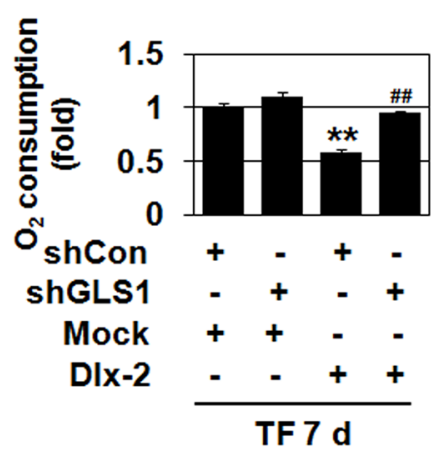
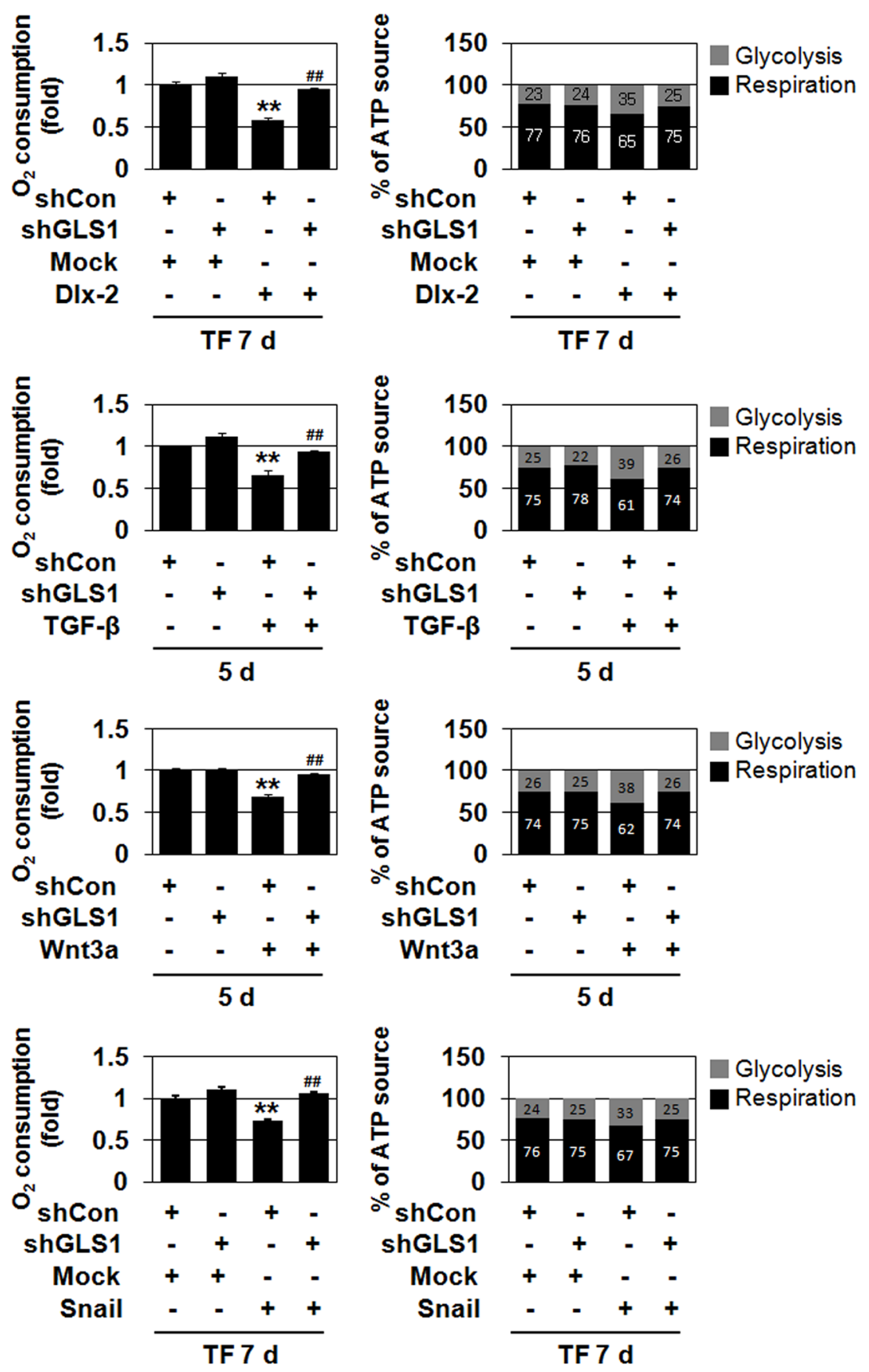

Figure 5: Gln metabolism is linked to TGF- $\beta$-, Wnt3a-, and Dlx-2/Snail-induced glycolytic switch and mitochondrial repression. A. MCF-7 cells were co-transfected with Dlx-2 and shGLS1. The cells were analyzed for Glc consumption, Lac production, mitochondrial respiration, and ATP source. ${ }^{* *} p<0.01$ versus Mock, ${ }^{*} p<0.01$ versus shCon. B, C. MCF-7 cells were transfected with shGLS1 and then treated with TGF- $\beta$ (B) or Wnt3a CM (C). The cells were analyzed for Glc consumption, Lac production, mitochondrial respiration, and ATP source. ${ }^{* *} p<0.01$ versus untreated, ${ }^{* \#} p<0.01$ versus shCon. D. MCF-7 cells were co-transfected with Snail and shGLS1. The cells were analyzed for Glc consumption, Lac production, mitochondrial respiration and ATP source. ${ }^{* *} p<0.01$ versus Mock, ${ }^{\# \#} p<0.01$ versus shCon. The amount of ATP produced by aerobic respiration (black bars) and glycolysis (gray bars) was calculated by measuring oxygen consumption and Lac production in the cells (right panels in A-D). All error bars represent SE. 
correlates positively with more advanced cancer stages and with poor prognosis in a variety of human cancer types [33-36]. In this study, we show that Dlx-2 induces EMT and regulates GLS1 expression and Gln metabolism, which increases tumor metastasis. The Dlx-2 target gene GLS1 (Figure 1) is important in Gln metabolism. GLS1 is a critical enzyme in a number of cancers [12-17]. GLS1 levels are increased in breast and prostate cancers and HCC tissues compared to matched normal tissues and positively correlate with both degree of malignancy and tumor grade [17, 18] (Figure 6). c-Myc-mediated repression of $\mathrm{miR}-23 \mathrm{a} / \mathrm{b}$ increases GLS1 levels $[12,13]$. In addition, the NF- $\kappa \mathrm{B}$ member p65 subunit suppresses the expression of miR-23a and directly inhibits GLS1 expression [42]. Xiang et al. (2015) showed that GLS1 mRNA is upregulated in primary human HCC compared to surrounding non-tumor tissue, and that, while $M Y C$ mRNA levels were also elevated in the tumors, they did not correlate directly with GLS1 mRNA levels [17]. This suggests that factors other than MYC may be involved in the upregulation of GLS1 mRNA levels in HCC. Here, GLS1 was induced by Dlx-2 (Figure 1). We showed that GLS1 expression was also upregulated by TGF- $\beta$ and Wnt in a Dlx-2-dependent, but Snail-independent, manner (Figure 1). Furthermore, Dlx-2 bound to the GLS1 promoter (Figure 1E), indicating that Dlx-2 induces GLS1 expression. Whether Dlx-2 binding is required for Dlx-2induced GLS1 activation remains to be elucidated through the use of transcriptional reporter assays combined with site-directed mutagenesis.

We showed that shGLS1 inhibits tumor growth and metastasis in vivo (Figure 2). shGLS1 and GLS-specific inhibitors, BPTES and 968, reduce the growth of several types of cancer cell xenografts $[10,14,17]$. However, the effects of shGLS1 and GLS1 inhibitors on metastasis have been not reported. Our results show an important role for the Dlx-2-GLS1/Gln metabolism cascade in tumor metastasis as well as in tumor growth.

Because EMT is an essential process in the initiation of metastasis [19-23], we investigated the effects of GLS1/Gln metabolism inhibition on EMT. Gln metabolism inhibition by shGLS1, Gln deprivation, and Gln metabolism inhibitors (DON, 968, BPTES) prevented Dlx-2-, TGF- $\beta$-, and Wnt-induced EMT, indicating that GLS1/Gln metabolism may be involved in Dlx-2/TGF- $\beta$ / Wnt-induced EMT (Figure 3, and Supplemental Figure 1 and 2). The Dlx-2-GLS1-Gln metabolism axis is thought to be an essential regulator of TGF- $\beta$ - and Wnt-induced EMT. Interestingly, shGLS1 also inhibited Snail-induced EMT, although GLS1 expression was not induced by Snail (Figure 3J-3L). Thus, the Dlx-2-GLS1-Gln axis may

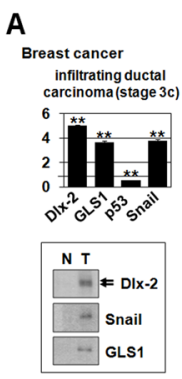

D

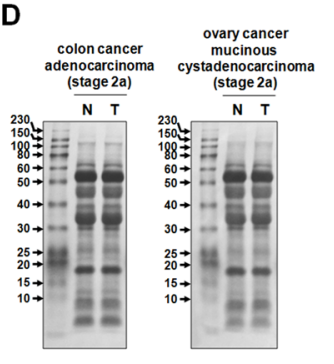

B Colon cancer adenocarcinoma
(stage 2a)
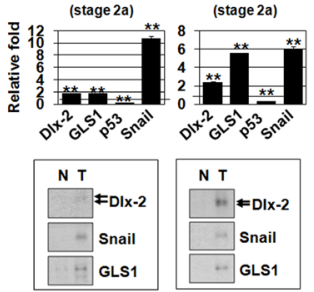

E

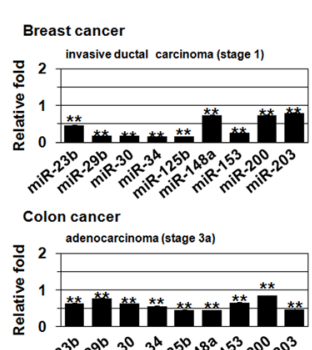

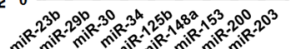

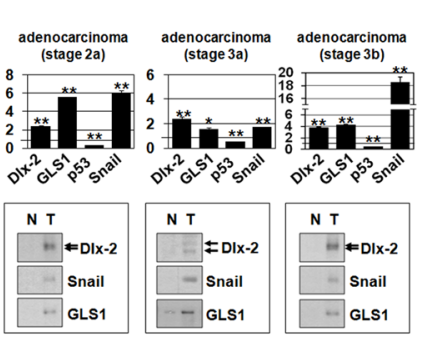

GLS1

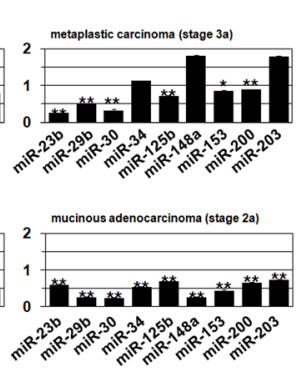

C

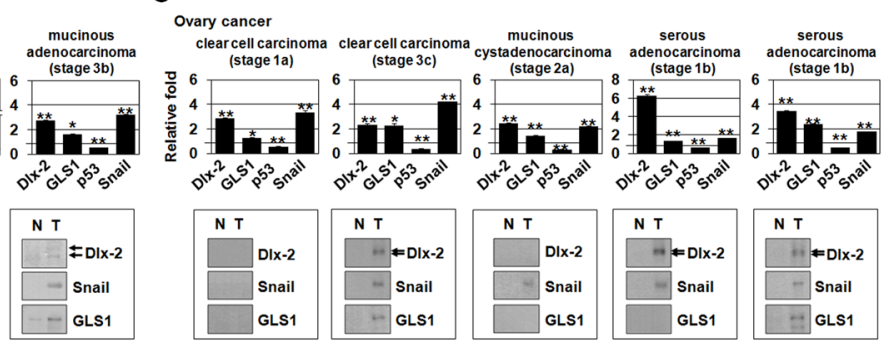

F

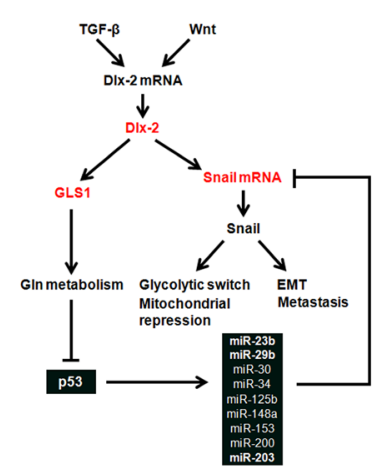

Figure 6: The expression of Dlx-2, GLS1, p53, Snail, and Snail-targeting miRNAs in human tumors. A-C. Real-time qrtPCR data showing expression of Dlx-2, GLS1, p53 and Snail mRNA, and immunoblotting for Dlx-2, Snail, and GLS1 protein in normal $(\mathrm{N})$ and tumor (T) tissues from the indicated tumor types and histological stages (TNM classification) of breast (A), colon (B) and ovarian cancer (C). D. Ponceau S-stained membranes showing loading of same amount of proteins. E. Real-time qrtPCR data of Snail-targeting miRNAs in normal $(\mathrm{N})$ and tumor $(\mathrm{T})$ tissues in the indicated tumor types. Relative levels of miRNAs were normalized to the corresponding normal tissues. ${ }^{*} p<0.05 ;{ }^{*} p<0.01$ versus matched normal $(\mathrm{N})$ tissues. All error bars represent SE. F. A schematic representation showing a new function of Dlx-2/GLS1-driven Gln metabolism that contributes to Snail-dependent EMT and glycolytic switch. For all immunoblotting images, cropped blots are shown. 
provide metabolic support for Snail-induced EMT, rather than acting as an upstream pathway.

Gln metabolism inhibition together with shGLS1 or Gln deprivation prevented Dlx-2-, TGF- $\beta-$, Wnt-, and Snail-induced glycolytic switch, indicating that Gln metabolism is closely linked to glycolytic switch (Figure 5, and Supplemental Figure 5). Recently, long-term exposure of cancer cells to acidic $\mathrm{pH}$ (resulting from glycolysis) has been shown to upregulate Gln metabolism to ensure intracellular $\mathrm{pH}$ homeostasis [43]. Thus, Gln and Glc metabolism influence each other in cancer cells.

Gln metabolism inhibition decreased Snail mRNA stability (Figure 3, and Supplemental Figure 3B). In addition, shDlx-2, shGLS1, Gln deprivation, and DON increased the expression of several Snail-targeting miRNAs (Table 1). Thus, the Dlx-2/GLS1/Gln metabolism pathway seems to increase Snail mRNA stability by regulating Snail-targeting miRNAs (Figure 6F).

We further showed that Gln metabolism inhibition increased levels of p53 protein and mRNA (Figure 4A4D). Recently, it was shown that Gln deprivation induced p53 through B55 $\alpha$ [44]. Here, shDlx-2, but not shSnail, increased p53 expression (Figure 4A, 4B, 4E and 4F). In addition, $\alpha-K G$ suppressed shDlx-2- and shGLS1-induced p53 expression (Figure 4A and 4B), indicating that p53 is downregulated by Dlx-2-driven Gln metabolism. Snailtargeting miRNA expression was upregulated by p53 (Table 1). Our results indicate that Snail mRNA stability is linked to p53 levels. Taken together, Gln metabolism inhibition prevented TGF- $\beta$-induced EMT and glycolytic switch by increasing Snail-targeting miRNA expression via p53, ultimately decreasing Snail stability (Figure 6F).

p53 is the strongest tumor suppressor gene, and almost all human cancers involve the loss of p53 function [45]. Dlx-2-induced Gln metabolism likely contributes to p53 reduction during tumor development. Gln deprivation induced p53 via ROS-dependent B55 $\alpha$ activation [44]. Thus, Gln metabolism inhibition may re-activate $\mathrm{p} 53$. In addition, TGF- $\beta$-induced EMT was more prominent in shp53-treated MCF-7 cells than in shCon-treated MCF-7 cells (Figure 4G and 4H). p53 activates the transcription of miR-200 and miR-34 family members [39, 46, 47]. Furthermore, loss of p53 function and p53 mutations promote cancer cell EMT by decreasing miRNA-34 levels in colon, breast, and lung carcinoma cells, which disinhibits Snail protein expression and activity [39].

We found that Gln metabolism inhibition also prevented Wnt3a-induced EMT and glycolytic switch (Figure 3G-3I and 5C, and Supplemental Figure 2C and 2D). TGF- $\beta$ induces Snail mRNA levels though Dlx2 activation. However, Wnt increased Snail levels in a Dlx-2-independent manner. Although the mechanisms by which TGF- $\beta$ and Wnt3a induce Snail expression differ, the effects of Gln metabolism inhibition on Wnt3a-induced EMT and glycolytic switch were similar. Wnt signaling induces EMT by activating an Axin2 pathway that stabilizes Snail. Axin2 stimulates EMT by acting as a chaperone for nuclear export of GSK3 $\beta$, the dominant kinase responsible for Snail protein turnover [48]. miR-34 directly suppresses Axin2 and other Wnt signaling molecules, including $\beta$-catenin and LEF1, in addition to Snail, ultimately suppressing EMT [39, 49]. Therefore, Gln metabolism inhibition seems to prevent Wnt3a-induced EMT and glycolytic switch through p53dependent expression of miR-34.

We further showed that Dlx-2 levels were also reduced by Gln metabolism inhibition (shGLS1, Gln deprivation or DON) in Dlx-2-overexpressing cells and TGF- $\beta$-treated cells (Figure $3 \mathrm{~B}, 3 \mathrm{E}$ and $3 \mathrm{~F}$, and Supplemental Figure 1B, 1E, 1J, 2B and 2H). We identified Snail-targeting miRNAs that might bind to the 3'-UTRs of Dlx-2 using miRanda, a target prediction program. Several miRNAs, including miR-23b and miR-203, were predicted to bind to the Dlx-2 3'-UTR (data not shown).

Our results showed that the Dlx-2/GLS1/Gln metabolism axis is involved in TGF- $\beta / \mathrm{Wnt} /$ Snail-induced EMT and glycolytic switch. Dlx-2 increased Snail mRNA stability by activating GLS1/Gln metabolism, which inhibited p53-dependent upregulation of Snail-targeting miRNAs (Figure 6F). Dlx-2 induces Snail gene expression [37]. Thus, Dlx-2 may induce Snail mRNA expression through two mechanisms; (i) activation of Snail mRNA transcription at an early time point, and (ii) increased Snail mRNA stability at a late time point.

Gln deprivation prevented Dlx-2-, TGF- $\beta-$, Wnt-, and Snail-induced EMT by reducing Snail mRNA levels through p53-dependent upregulation of Snail-targeting miRNAs. Gln activates the highly conserved kinase mammalian target of rapamycin complex 1 (mTORC1), which stimulates protein translation and cell growth; thus, Gln deprivation strongly suppresses growth by inhibiting mTORC1 in several types of cells [50-52]. Gln deprivation also inhibits global protein translation by reducing GCN2 protein kinase-mediated phosphorylation of the translation initiation factor eIF $2 \alpha[53,54]$. Many studies have shown that mTOR signaling activates EMT, cancer invasion, and metastasis [55-57]. Although genetic and pharmacologic inhibition of mTORC1 triggers EMT in normal immortalized human epithelial cell lines and primary epithelial cells [58], mTORC1 inhibition represses EMT in colon and breast cancer cells [59]. This difference in the effect of mTORC1 inhibition on EMT is likely due to differences in the mutational status of the cells [59]. Cai et al. (2014) showed that mTORC1 induces phosphorylation of the cap-dependent translation repressor 4E-BP1, which leads to its dissociation from eIF4E and formation of the translation initiation complex. This results in cap-dependent activation of Snail translation and the induction of EMT in cancer cells [59]. mTORC1/4EBP1-mediated Snail translation may also contribute to Dlx-2/Gln metabolism-induced Snail expression. Whether 
Dlx-2/GLS1/Gln metabolism activates mTORC1 signaling requires further investigation.

Oncogenic metabolism, including Gln metabolism, has been suggested to confer growth advantages to cancer cells by providing biosynthetic precursors [2, 4-8]. We propose that, in addition to its well-described role in supporting tumor growth by providing metabolites, Gln metabolism may also contribute to tumor EMT, metastasis, and progression. Metastasis is a complex process and includes detachment of tumor cells from the primary site. The loss of cell-matrix interactions induces anoikis, and resistance to anoikis is a prerequisite for tumor metastasis [60, 61]. Snail induces a glycolytic switch and suppresses mitochondrial oxidative metabolism [30], which may contribute to anoikis resistance and metastasis by preventing excess ROS generation. Thus, the Dlx-2/GLS1 cascade may promote tumor metastasis by increasing Snail-mediated anoikis resistance in addition to EMT. Our results suggest that GLS1 may be a potential therapeutic target for the prevention of metastasis and tumor progression.

\section{MATERIALS AND METHODS}

\section{Cell culture}

MCF-7, Madin Darby Canine Kidney (MDCK), and L cells from the American Type Culture Collection (ATCC) were cultured under established conditions [29]. Wnt3a-secreting L cells and HCT116 cells were provided by Dr. Min DS and Dr. Kim YJ (Pusan National University, Pusan, Korea), respectively. The Gln deprivation experiment was performed by applying EMEM without Gln (GIBCO, Carlsbad, CA, USA). Recombinant TGF- $\beta$ (R\&D Systems, MN, USA) was applied to cells at a concentration of $10 \mathrm{ng} / \mathrm{ml} .40 \mu \mathrm{M}$ DON (Sigma, St. Louis, MO, USA), 5 M 968 (Calbiochem, San Diego, CA, USA), $10 \mu \mathrm{M}$ BPTES (Sigma), or $1 \mathrm{mM}$ dimethyl$\alpha$-ketoglutarate $(\alpha-\mathrm{KG}$; Sigma) were also applied to cells.

\section{Transfection and short hairpin RNA (shRNA) interference}

The expression vectors pCAGGS-Dlx-2 (provided by Dr. John L.R. Rubenstein, University of California at San Francisco) and pCR3.1-Snail-Flg (provided by J.I. Yook, Yonsei University, Korea) were transfected into MCF-7, MDCK, and HCT116 cells using jetPEI (Polyplus transfection, SA, USA). pSUPER vectors for shRNA against control, Dlx-2, Snail, GLS1, Smad2, Smad3, Smad4, $\beta$-catenin, TCF4, Axin1, Axin2, and p53 (abbreviations; shCon, shDlx-2, etc.) were produced and transfected as described previously [29]. shRNA target sequences are listed in Supplementary Table S1.

\section{Immunoblotting, real-time qrtPCR, immunofluorescence (IF) staining, and chromatin immunoprecipitation (ChIP) assay}

Immunoblotting, real-time qrtPCR, IF and ChIP assay were performed as described previously [29, 33]. Primers for real-time qrtPCR and ChIP assays, and putative Dlx-2 binding sites in promoter region are listed in Supplementary Table S2 and S3.

\section{Assays for mitochondrial respiration, Glc consumption, Lac production, and ATP production}

Mitochondrial respiration was measured as described previously [29, 62]. Glc, Lac and intracellular ATP levels in the media were determined using a Glc oxidation assay kit (Sigma, MO, USA), a colorimetric and fluorescence-based Lac assay kit (BioVision, California, USA), and an ATP Bioluminescence Assay kit (Roche, Switzerland), respectively, according to the manufacturers' instructions. Levels of Glc, Lac, and intracellular ATP were normalized to protein concentrations. Levels of ATP produced by aerobic respiration and glycolysis were determined by measuring Lac production and oxygen consumption $[29,63]$.

\section{ACKNOWLEDGMENTS}

This work was supported by the National Research Foundation of Korea (NRF) grant funded by the Korean government (MSIP) (No. 2011-0011084, 2013M2B2A9A03050902 and 2012R1A1A2044246) and by a grant from the National R\&D Program for Cancer Control, Ministry of Health and Welfare, Republic of Korea (1320040). We thank Drs. K.L. Jang, Y. H. Moon and D. S. Min for providing their real-time qrtPCR machines.

\section{CONFLICTS OF INTEREST}

The authors declare no conflicts of interest.

\section{Author contributions}

S.Y. Lee, H.M. Jeon and H.S. Kang conceived and designed the project. S.Y. Lee, H.M. Jeon, M.K. Ju and E.K. Jeong performed the experiments. S.Y. Lee, H.M. Jeon, M.K. Ju, E.K. Jeong, C.H. Kim, H.G. Park, S.I. Han and H.S. Kang analyzed and interpreted the data. S.Y. Lee, H.M. Jeon, E.K. Jeong and H.S. Kang wrote the manuscript. H.S. Kang supervised the project. 


\section{REFERENCES}

1. Hsu PP, Sabatini DM. Cancer cell metabolism: Warburg and beyond. Cell. 2008; 134: 703-707.

2. Vander Heiden MG, Cantley LC, Thompson CB. Understanding the Warburg effect: the metabolic requirements of cell proliferation. Science. 2009; 324: 1029-1033.

3. Warburg O. On the origin of cancer cells. Science. 1956; 123: 309-314.

4. Cairns RA, Harris IS, Mak TW. Regulation of cancer cell metabolism. Nat Rev Cancer. 2011; 11: 85-95.

5. Dang CV. Links between metabolism and cancer. Genes Dev. 2012; 26: 877-890.

6. Schulze A, Harris AL. How cancer metabolism is tuned for proliferation and vulnerable to disruption. Nature. 2012; 491: 364-373.

7. Finley LW, Zhang J, Ye J, Ward PS, Thompson CB. SnapShot: cancer metabolism pathways. Cell Metab. 2013; 17: 466-466 e462.

8. Boroughs LK, DeBerardinis RJ. Metabolic pathways promoting cancer cell survival and growth. Nat Cell Biol. 2015; 17: 351-359.

9. Owen OE, Kalhan SC, Hanson RW. The key role of anaplerosis and cataplerosis for citric acid cycle function. J Biol Chem. 2002; 277: 30409-30412.

10. Le A, Lane AN, Hamaker M, Bose S, Gouw A, Barbi J, Tsukamoto T, Rojas CJ, Slusher BS, Zhang H, Zimmerman LJ, Liebler DC, Slebos RJ et al. Glucose-independent glutamine metabolism via TCA cycling for proliferation and survival in B cells. Cell Metab. 2012; 15: 110-121.

11. Son J, Lyssiotis CA, Ying H, Wang X, Hua S, Ligorio M, Perera RM, Ferrone CR, Mullarky E, Shyh-Chang N, Kang Y, Fleming JB, Bardeesy N et al. Glutamine supports pancreatic cancer growth through a KRAS-regulated metabolic pathway. Nature. 2013; 496: 101-105.

12. Wise DR, DeBerardinis RJ, Mancuso A, Sayed N, Zhang XY, Pfeiffer HK, Nissim I, Daikhin E, Yudkoff M, McMahon SB, Thompson CB. Myc regulates a transcriptional program that stimulates mitochondrial glutaminolysis and leads to glutamine addiction. Proc Natl Acad Sci U S A. 2008; 105: 18782-18787.

13. Gao P, Tchernyshyov I, Chang TC, Lee YS, Kita K, Ochi T, Zeller KI, De Marzo AM, Van Eyk JE, Mendell JT, Dang CV. c-Myc suppression of miR-23a/b enhances mitochondrial glutaminase expression and glutamine metabolism. Nature. 2009; 458: 762-765.

14. Wang JB, Erickson JW, Fuji R, Ramachandran S, Gao P, Dinavahi R, Wilson KF, Ambrosio AL, Dias SM, Dang CV, Cerione RA. Targeting mitochondrial glutaminase activity inhibits oncogenic transformation. Cancer Cell. 2010; 18: 207-219.

15. Seltzer MJ, Bennett BD, Joshi AD, Gao P, Thomas AG, Ferraris DV, Tsukamoto T, Rojas CJ, Slusher BS, Rabinowitz JD, Dang CV, Riggins GJ. Inhibition of glutaminase preferentially slows growth of glioma cells with mutant IDH1. Cancer Res. 2010; 70: 8981-8987.

16. Hensley CT, Wasti AT, DeBerardinis RJ. Glutamine and cancer: cell biology, physiology, and clinical opportunities. J Clin Invest. 2013; 123: 3678-3684.

17. Xiang Y, Stine ZE, Xia J, Lu Y, O’Connor RS, Altman BJ, Hsieh AL, Gouw AM, Thomas AG, Gao P, Sun L, Song L, Yan B et al. Targeted inhibition of tumor-specific glutaminase diminishes cell-autonomous tumorigenesis. J Clin Invest. 2015; 125: 2293-2306.

18. Cassago A, Ferreira AP, Ferreira IM, Fornezari C, Gomes ER, Greene KS, Pereira HM, Garratt RC, Dias SM, Ambrosio AL. Mitochondrial localization and structurebased phosphate activation mechanism of Glutaminase C with implications for cancer metabolism. Proc Natl Acad Sci U S A. 2012; 109: 1092-1097.

19. De Craene B, Berx G. Regulatory networks defining EMT during cancer initiation and progression. Nat Rev Cancer. 2013; 13: 97-110.

20. Tsai JH, Yang J. Epithelial-mesenchymal plasticity in carcinoma metastasis. Genes Dev. 2013; 27: 2192-2206.

21. Quail DF, Joyce JA. Microenvironmental regulation of tumor progression and metastasis. Nat Med. 2013; 19: 1423-1437.

22. Puisieux A, Brabletz T, Caramel J. Oncogenic roles of EMT-inducing transcription factors. Nat Cell Biol. 2014; 16: 488-494.

23. Weber GF. Why does cancer therapy lack effective antimetastasis drugs? Cancer Lett. 2013; 328: 207-211.

24. Peinado H, Olmeda D, Cano A. Snail, Zeb and bHLH factors in tumour progression: an alliance against the epithelial phenotype? Nat Rev Cancer. 2007; 7: 415-428.

25. Wang Y, Shi J, Chai K, Ying X, Zhou BP. The Role of Snail in EMT and Tumorigenesis. Curr Cancer Drug Targets. 2013; 13: 963-972.

26. De Craene B, Denecker G, Vermassen P, Taminau J, Mauch C, Derore A, Jonkers J, Fuchs E, Berx G. Epidermal Snail expression drives skin cancer initiation and progression through enhanced cytoprotection, epidermal stem/progenitor cell expansion and enhanced metastatic potential. Cell Death Differ. 2014; 21: 310-320.

27. Anastas JN, Moon RT. WNT signalling pathways as therapeutic targets in cancer. Nat Rev Cancer. 2013; 13: 11-26.

28. Pickup M, Novitskiy S, Moses HL. The roles of TGFbeta in the tumour microenvironment. Nat Rev Cancer. 2013; 13: 788-799.

29. Lee SY, Jeon HM, Ju MK, Kim CH, Yoon G, Han SI, Park HG, Kang HS. Wnt/Snail Signaling Regulates Cytochrome c Oxidase and Glucose Metabolism. Cancer Res. 2012; 72 : 3607-3617.

30. Dong C, Yuan T, Wu Y, Wang Y, Fan TW, Miriyala S, Lin Y, Yao J, Shi J, Kang T, Lorkiewicz P, St Clair D, Hung MC et al. Loss of FBP1 by Snail-mediated repression 
provides metabolic advantages in basal-like breast cancer. Cancer Cell. 2013; 23: 316-331.

31. Merlo GR, Zerega B, Paleari L, Trombino S, Mantero S, Levi G. Multiple functions of Dlx genes. Int J Dev Biol. 2000; 44: 619-626.

32. Panganiban G, Rubenstein JL. Developmental functions of the Distal-less/Dlx homeobox genes. Development. 2002; 129: 4371-4386.

33. Lee SY, Jeon HM, Kim CH, Ju MK, Bae HS, Park HG, Lim SC, Han SI, Kang HS. Homeobox gene Dlx-2 is implicated in metabolic stress-induced necrosis. Mol Cancer. 2011; 10: 113.

34. Yilmaz M, Maass D, Tiwari N, Waldmeier L, Schmidt P, Lehembre F, Christofori G. Transcription factor Dlx2 protects from TGFbeta-induced cell-cycle arrest and apoptosis. Embo J. 2011; 30: 4489-4499.

35. Tang P, Huang H, Chang J, Zhao GF, Lu ML, Wang Y. Increased expression of DLX2 correlates with advanced stage of gastric adenocarcinoma. World J Gastroenterol. 2013; 19: 2697-2703.

36. Yan ZH, Bao ZS, Yan W, Liu YW, Zhang CB, Wang HJ, Feng Y, Wang YZ, Zhang W, You G, Zhang QG, Jiang T. Upregulation of DLX2 confers a poor prognosis in glioblastoma patients by inducing a proliferative phenotype. Curr Mol Med. 2013; 13: 438-445.

37. Lee SY, Jeon HM, Ju MK, Jeong EK, Kim CH, Yoo MA, Park HG, Han SI, Kang HS. Dlx-2 is implicated in TGFbeta- and Wnt-induced epithelial-mesenchymal, glycolytic switch, and mitochondrial repression by Snail activation. Int J Oncol. 2015; 46: 1768-1780.

38. Neve RM, Chin K, Fridlyand J, Yeh J, Baehner FL, Fevr T, Clark L, Bayani N, Coppe JP, Tong F, Speed T, Spellman $\mathrm{PT}$, DeVries $\mathrm{S}$ et al. A collection of breast cancer cell lines for the study of functionally distinct cancer subtypes. Cancer Cell. 2006; 10: 515-527.

39. Kim NH, Kim HS, Li XY, Lee I, Choi HS, Kang SE, Cha SY, Ryu JK, Yoon D, Fearon ER, Rowe RG, Lee S, Maher CA et al. A p53/miRNA-34 axis regulates Snail1-dependent cancer cell epithelial-mesenchymal transition. J Cell Biol. 2011; 195: 417-433.

40. Reid MA, Kong M. Dealing with hunger: Metabolic stress responses in tumors. J Carcinog. 2013; 12: 17.

41. Lopez-Diaz FJ, Gascard P, Balakrishnan SK, Zhao J, Del Rincon SV, Spruck C, Tlsty TD, Emerson BM. Coordinate transcriptional and translational repression of $\mathrm{p} 53$ by TGFbeta1 impairs the stress response. Mol Cell. 2013; 50: 552-564.

42. Rathore MG, Saumet A, Rossi JF, de Bettignies C, Tempe D, Lecellier CH, Villalba M. The NF-kappaB member p65 controls glutamine metabolism through miR-23a. Int $\mathrm{J}$ Biochem Cell Biol. 2012; 44: 1448-1456.

43. Corbet C, Draoui N, Polet F, Pinto A, Drozak X, Riant O, Feron O. The SIRT1/HIF2alpha axis drives reductive glutamine metabolism under chronic acidosis and alters tumor response to therapy. Cancer Res. 2014; 74: 5507-5519.

44. Reid MA, Wang WI, Rosales KR, Welliver MX, Pan M, Kong M. The B55alpha subunit of PP2A drives a p53-dependent metabolic adaptation to glutamine deprivation. Mol Cell. 2013; 50: 200-211.

45. Vousden KH, Prives C. P53 and prognosis: new insights and further complexity. Cell. 2005; 120: 7-10.

46. Chang CJ, Chao CH, Xia W, Yang JY, Xiong Y, Li CW, Yu WH, Rehman SK, Hsu JL, Lee HH, Liu M, Chen CT, $\mathrm{Yu}$ D et al. p53 regulates epithelial-mesenchymal transition and stem cell properties through modulating miRNAs. Nat Cell Biol. 2011; 13: 317-323.

47. Hahn S, Jackstadt R, Siemens H, Hunten S, Hermeking H. SNAIL and miR-34a feed-forward regulation of ZNF281/ ZBP99 promotes epithelial-mesenchymal transition. Embo J. 2013; 32: 3079-3095.

48. Yook JI, Li XY, Ota I, Hu C, Kim HS, Kim NH, Cha SY, Ryu JK, Choi YJ, Kim J, Fearon ER, Weiss SJ. A WntAxin2-GSK3beta cascade regulates Snaill activity in breast cancer cells. Nat Cell Biol. 2006; 8: 1398-1406.

49. Kim NH, Cha YH, Kang SE, Lee Y, Lee I, Cha SY, Ryu JK, Na JM, Park C, Yoon HG, Park GJ, Yook JI, Kim HS. p53 regulates nuclear GSK-3 levels through miR-34mediated Axin 2 suppression in colorectal cancer cells. Cell Cycle. 2013; 12: 1578-1587.

50. Nicklin P, Bergman P, Zhang B, Triantafellow E, Wang H, Nyfeler B, Yang H, Hild M, Kung C, Wilson C, Myer VE, MacKeigan JP, Porter JA et al. Bidirectional transport of amino acids regulates mTOR and autophagy. Cell. 2009; 136: 521-534.

51. Sengupta S, Peterson TR, Sabatini DM. Regulation of the mTOR complex 1 pathway by nutrients, growth factors, and stress. Mol Cell. 2010; 40: 310-322.

52. Duran RV, Oppliger W, Robitaille AM, Heiserich L, Skendaj R, Gottlieb E, Hall MN. Glutaminolysis activates Rag-mTORC1 signaling. Mol Cell. 2012; 47: 349-358.

53. Scorsone KA, Panniers R, Rowlands AG, Henshaw EC. Phosphorylation of eukaryotic initiation factor 2 during physiological stresses which affect protein synthesis. J Biol Chem. 1987; 262: 14538-14543.

54. Qing G, Li B, Vu A, Skuli N, Walton ZE, Liu X, Mayes PA, Wise DR, Thompson CB, Maris JM, Hogarty MD, Simon MC. ATF4 regulates MYC-mediated neuroblastoma cell death upon glutamine deprivation. Cancer Cell. 2012; 22: 631-644.

55. Lamouille S, Derynck R. Cell size and invasion in TGFbeta-induced epithelial to mesenchymal transition is regulated by activation of the mTOR pathway. J Cell Biol. 2007; 178: 437-451.

56. Gulhati P, Bowen KA, Liu J, Stevens PD, Rychahou PG, Chen M, Lee EY, Weiss HL, O'Connor KL, Gao T, Evers BM. mTORC1 and mTORC2 regulate EMT, motility, and 
metastasis of colorectal cancer via RhoA and Rac1 signaling pathways. Cancer Res. 2011; 71: 3246-3256.

57. Hsieh AC, Liu Y, Edlind MP, Ingolia NT, Janes MR, Sher A, Shi EY, Stumpf CR, Christensen C, Bonham MJ, Wang $\mathrm{S}$, Ren P, Martin M et al. The translational landscape of mTOR signalling steers cancer initiation and metastasis. Nature. 2012; 485: 55-61.

58. Mikaelian I, Malek M, Gadet R, Viallet J, Garcia A, GirardGagnepain A, Hesling C, Gillet G, Gonzalo P, Rimokh $\mathrm{R}$, Billaud M. Genetic and pharmacologic inhibition of mTORC1 promotes EMT by a TGF-beta-independent mechanism. Cancer Res. 2013; 73: 6621-6631.

59. Cai W, Ye Q, She QB. Loss of 4E-BP1 function induces EMT and promotes cancer cell migration and invasion via cap-dependent translational activation of snail. Oncotarget. 2014; 5: 6015-6027. doi: 10.18632/oncotarget.2109
60. Paoli P, Giannoni E, Chiarugi P. Anoikis molecular pathways and its role in cancer progression. Biochim Biophys Acta. 2013; 1833: 3481-3498.

61. Lu J, Tan M, Cai Q. The Warburg effect in tumor progression: mitochondrial oxidative metabolism as an antimetastasis mechanism. Cancer Lett 2015; 28: 156-164.

62. Yoon YS, Lee JH, Hwang SC, Choi KS, Yoon G. TGF betal induces prolonged mitochondrial ROS generation through decreased complex IV activity with senescent arrest in Mv1Lu cells. Oncogene. 2005; 24: 1895-1903.

63. Sariban-Sohraby S, Magrath IT, Balaban RS. Comparison of energy metabolism in human normal and neoplastic (Burkitt's lymphoma) lymphoid cells. Cancer Res. 1983; 43: 4662-4664. 